\title{
Une plateforme et des modèles pour la scénarisation coopérative de modules pédagogiques
}

\author{
A platform and models for cooperative scenarization \\ of learning modules
}

\section{Entorno y modelos para la escenarización cooperativa de módulos de formación}

\author{
Patrick Etcheverry, Christophe Marquesuzaà, Philippe Lopistéguy, Pantxika Dagorret, \\ Thierry Nodenot \\ Univ Pau \& Pays Adour / E2S UPPA, Laboratoire d'Informatique de l'Université de Pau et des Pays de \\ l'Adour, EA3000, France \\ prenom.nom@iutbayonne.univ-pau.fr
}

Marta Toribio Fontenla

IUT de Bayonne et du Pays Basque, France

marta.toribio@iutbayonne.univ-pau.fr

RÉSUMÉ

Cet article s'intéresse à la scénarisation de modules d'enseignement et à la démarche permettant de mener cette scénarisation. Le point de départ de ces travaux est une expérience menée pour mettre en ligne une formation à distance diplômante. Nous avons souhaité capitaliser l'expérience de scénarisation mise en œuvre au cours de ce travail. À cette fin, cet article présente les modèles que nous avons élaborés pour spécifier un scénario ainsi que la démarche à conduire pour mener le travail de scénarisation de manière coopérative. Ces modèles ont été intégrés au sein d'une plateforme Web de scénarisation permettant à des enseignants et des ingénieurs pédagogiques de coopérer pour spécifier des scénarios qui pourront être exportés sur le LMS Moodle.

Mots-clés : scénarisation pédagogique, scénarisation coopérative, modèles de scénario, plateforme de scénarisation, Moodle 
This paper focuses on the scenarization of learning/teaching modules and on the process carried out to elaborate pedagogical scenarios. This work starting point is an experience conducted to create online training courses leading to a universitary diploma. We aim at capitalizing the scenarization skills deployed during this experience. To this end, this paper presents the models elaborated in order to specify not only a scenario but also the underlying scenarization approach. These models have been integrated in an environment allowing pedagogical engineers and teachers to cooperatively specify courses and to put them online on the Moodle platform.

Keywords: pedagogical scenarization, cooperative scenarization, scenario models, scenarization platform, Moodle

RESUMEN

Este artículo se centra en el diseño y escenarización de módulos de formación y en el proceso que guía su diseño. El punto de partida de este trabajo es una experiencia llevada a cabo para la creación de una formación en línea que otorga un diploma universitario. Nuestro equipo ha querido capitalizar la experiencia de escenarización utilizada durante este trabajo. Por ello presentamos los modelos que hemos elaborado para diseñar un escenario y el procedimiento de escenarización colaborativa. Los modelos producidos han sido integrados en una plataforma Web de escenarización, permitiendo la colaboración entre docentes e ingenieros pedagógicos para diseñar escenarios que podrán exportarse e integrarse en el LMS Moodle.

Palabras clave: escenarización pedagógica, escenarización colaborativa, modelos de escenario, entorno de escenarización, Moodle

\section{Introduction}

Les compétences numériques prennent une part croissante, que ce soit dans le quotidien du grand public, dans les entreprises, ou encore dans le domaine éducatif, de l'école primaire à l'Université. Dans le privé, par exemple en France chez Orange et ses 100000 salariés, l'objectif est de réaliser $50 \%$ des formations internes par MOOC (https://bit.ly/2W/13Rg) 1.

\footnotetext{
${ }^{1}$ https://www.journaldunet.com/management/ressources-humaines/1195759-interview-drh-orange/
} 
Le 5 mars 2018, Muriel Pénicaud, la ministre du Travail a dévoilé les grandes lignes de son projet de réforme de la formation professionnelle pour faciliter l'accès à la formation (https://bit.ly/2HsHIll4) ${ }^{2}$. Elle a annoncé qu'elle mettrait en avant les MOOC qui pourraient devenir massivement éligibles au compte personnel de formation (CPF). Le CPF permet à toute personne, salariée ou demandeur d'emploi, de suivre, à son initiative, une action de formation. II accompagne son titulaire dès l'entrée dans la vie professionnelle, tout au long de sa carrière jusqu'au départ en retraite (https://bit.ly/2IMrtYv) ${ }^{3}$. Les MOOC concernés par cette annonce seront ainsi recensés sur une plateforme en ligne qui obligerait leurs concepteurs à fournir un parcours pédagogique de qualité qui serait contrôlé par une agence de niveau national.

Dans le monde universitaire, les enseignants disposent de compétences reconnues et d'une longue expérience dans la création de cours en présentiel. Désormais, de nombreux établissements proposent un espace numérique de travail permettant, a minima, de mettre à disposition des apprenants des ressources numériques utilisées dans des séquences " traditionnelles » d'enseignement. Elles peuvent être utilisées avant les cours, par exemple dans un apprentissage en pédagogie inversée, pendant les cours, pour accéder à des supports non photocopiés, ou encore après les cours, pour réviser. La formation hybride intègre des dispositifs articulant à des degrés divers des phases de formation en présentiel et des phases de formation à distance, soutenues par un environnement technologique comme une plateforme de formation (Charlier et al., 2006). Ces formations hybrides permettent de lisser le temps de formation, mais pas de le contracter. Or, les formes d'enseignement, telles que les MOOC, SPOC... visent à s'adapter aux besoins de publics non disponibles pour de la formation en présentiel. II s'agit alors d'accompagner les enseignants dans le changement de leurs pratiques pédagogiques et c'est une des missions centrales des ingénieurs pédagogiques.

Notre proposition consiste à offrir une méthodologie et un environnement support permettant à un ingénieur pédagogique d'accompagner des enseignants dans la production de modules de formation hybride. Les retombées espérées sont multiples. Pour les apprenants, il s'agit d'améliorer leur motivation et de diversifier leurs modes d'apprentissage. Pour les enseignants, il s'agit d'élargir leur pratique pédagogique par le biais de la diversification des modes de transmission. Pour les institutions délivrant les modules d'apprentissage, il s'agit d'être performant dans la mise en place et dans le retour sur la montée en connaissances et compétences de tous les acteurs impliqués.

Le plan de cet article comprend les parties suivantes. Dans la section 2 nous présentons un retour d'expérience sur une démarche de scénarisation utilisée pour mettre en place une formation diplômante en ligne. Ce cas d'utilisation nous permet, dans la section 3, de positionner nos travaux vis-à-vis de l'état de l'art dans les domaines des scénarios pédagogiques et des méthodologies de scénarisation. La section 4 détaille les modèles que nous proposons afin de supporter ces deux aspects. Dans la section 5 , nous présentons une plateforme mettant en œuvre les propositions avancées pour supporter la conception coopérative d'enseignements hybrides. Dans la section 6, nous abordons sous forme de discussion les solutions envisagées pour gérer la dimension coopérative de la scénarisation. Enfin, nous concluons cet article en ouvrant des perspectives sur le travail à poursuivre.

\footnotetext{
${ }^{2}$ https://www.journaldunet.com/management/formation/1207757-reforme-de-la-formation-professionnelle-ce-qu-attendent-les-concepteurs-de$\underline{\mathrm{moocl}}$

${ }^{3}$ http://www.le-compte-personnel-formation.com/qu-est-ce-que-le-compte-personnel-de-formation-cpf/
} 


\section{Retour d'expérience sur une démarche de scénarisation}

Notre expérience a été menée dans le cadre de la création du diplôme universitaire "Administration des Systèmes et Réseaux » (DU ASR), en modalité à distance et en langue espagnole. Les publics visés sont des apprenants adultes vivant et travaillant en Amérique latine et en reconversion professionnelle. Leur objectif est d'acquérir des connaissances et des compétences dans le domaine des systèmes et réseaux afin d'exercer ensuite un métier de technicien supérieur.

Les formateurs ayant participé à la construction des modules de formation appartiennent à divers organismes d'enseignement supérieur en France (IUT de Bayonne et du Pays Basque), Espagne (Universidad del País Vasco / Euskal Herriko Unibertsitatea - UPV/EHU), Argentine (ITU Mendoza), Chili (CEDUC Universidad Católica del Norte) et Colombie (Universidad Tecnológica de Pereira).

La démarche de scénarisation ayant permis la création des modules en ligne constituant cette formation a été élaborée par un ingénieur pédagogique qui avait pour mission de coordonner 18 enseignants responsables de ces modules de 30 à 60 heures de travail étudiant réalisées en autonomie et à distance. La démarche de l'ingénieur pédagogique comprend trois étapes principales :

1) Prise de contact et identification de l'intention du cours;

2) Établissement d'un descriptif synthétique des chapitres composant le cours;

3) Élaboration du scénario détaillé décrivant le contenu de chaque chapitre.

La première étape prend la forme d'une prise de contact matérialisée par un questionnaire de 50 questions à destination de l'auteur du cours pour que l'ingénieur pédagogique identifie les pratiques de scénarisation pédagogique qui caractérisent la façon de faire de l'enseignant. Le questionnaire permet aussi d'identifier la relation de l'enseignant vis-à-vis de la formation ouverte et à distance (FOAD), à savoir quelles sont ses connaissances et pratiques personnelles. L'objectif pour l'ingénieur pédagogique est d'adapter la démarche de scénarisation en fonction du niveau de maîtrise de l'enseignant relativement à la FOAD. Ce questionnaire peut faire l'objet de plusieurs allers-retours entre l'enseignant et l'ingénieur pédagogique afin que ce dernier puisse cerner les pratiques pédagogiques non forcément exprimées lors du premier échange.

La deuxième étape concerne la structure générale du cours. L'ingénieur pédagogique fournit un formulaire commenté, détaillé et exemplifié que l'enseignant va devoir renseigner pour décrire le module à mettre en ligne. Les clauses du formulaire visent à détailler le public cible et les prérequis, les connaissances et compétences à acquérir, le matériel et les éventuels logiciels nécessaires, les méthodes et moyens d'évaluation envisagés, et une première liste de séquences pédagogiques (chapitres).

Sur la base du descriptif synthétique précédent, la troisième et dernière étape détaille le scénario constituant le cours. II s'agit :

- d'établir un découpage de chaque chapitre en activités dont les objectifs pédagogiques cumulés couvrent l'objectif du chapitre;

- de définir les ressources pédagogiques à médiatiser (ou les identifier si elles existent) qui seraient utiles à chaque activité;

- de s'accorder sur l'obtention et la livraison desdites ressources. 
Un extrait des documents produits à chaque étape peut être consulté à l'URL suivante : https://goo.gl/nYnzay

Cette tâche de scénarisation prend la forme d'un processus itératif de spécification se traduisant par plusieurs échanges durant lesquels l'ingénieur pédagogique apporte son expertise et des préconisations permettant d'aboutir à un scénario pédagogique de qualité et qui pourra être transposé sur une plateforme d'apprentissage (Moodle dans le cadre de l'expérience du DU ASR).

La démarche de scénarisation employée pour mettre au point les modules pédagogiques du DU ASR offre un cadre qui a permis d'élaborer à distance des modules d'enseignement avec des enseignants répartis sur plusieurs pays et avec des pratiques différentes. Les premiers modules de formation ont ainsi été mis en ligne en septembre 2015 et depuis mars 2017, les 11 modules (Figure 1) composant la formation ont été entièrement scénarisés et mis en ligne (https://bit.ly/2u7YDEO) ${ }^{5}$.

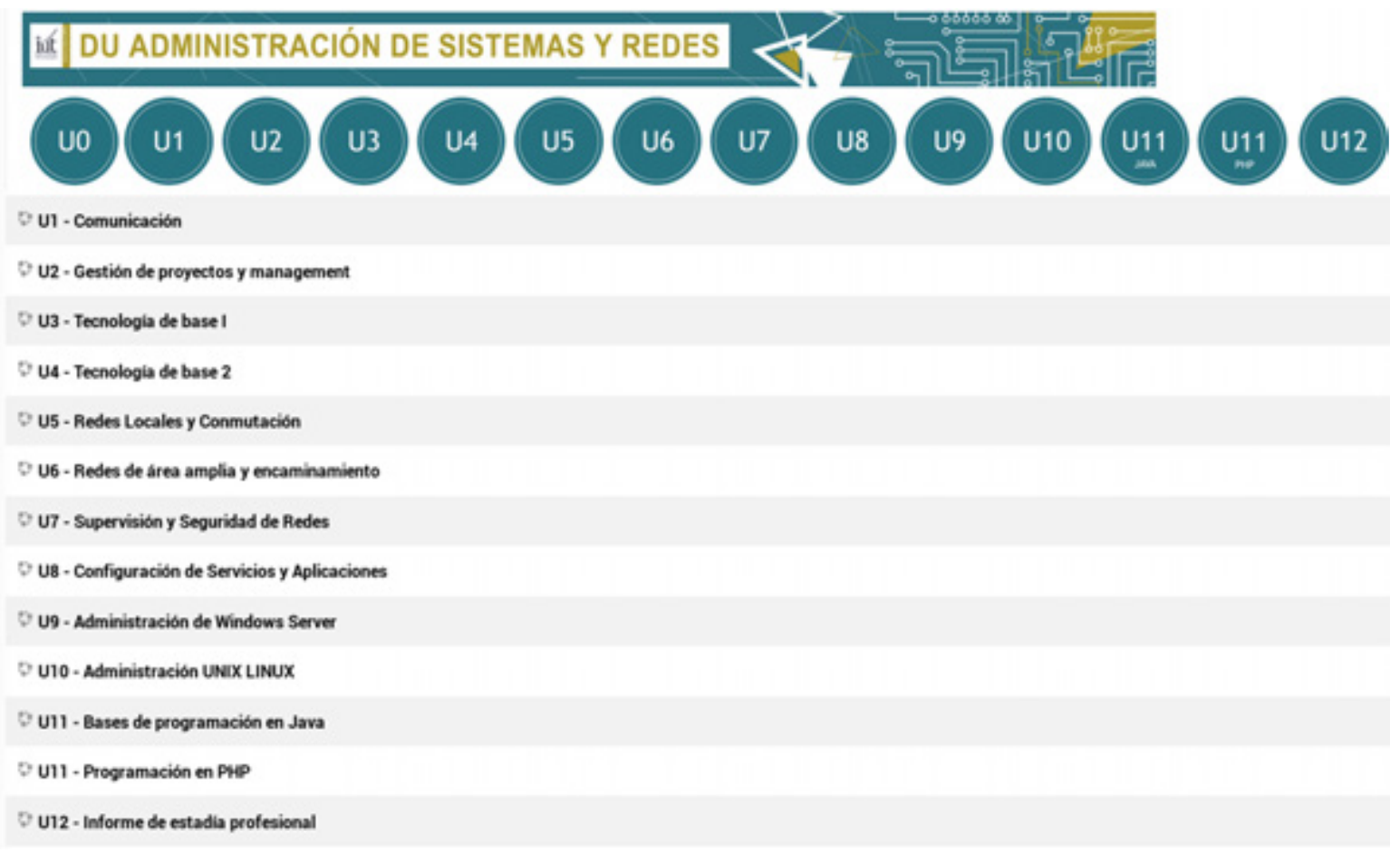

Figure 1. Modules scénarisés sur Moodle pour le DU ASR

Malgré ces résultats satisfaisants, le travail réalisé pour la mise en place cette formation permet de dégager plusieurs points améliorables :

- Les formulaires actuellement utilisés à chaque étape (fichiers MS Word et Excel échangés par mail) se prêtent mal à une scénarisation coopérative qui nécessite plusieurs échanges (entre l'enseignant et l'ingénieur pédagogique, mais aussi parfois entre les enseignants lorsque le module est conçu à plusieurs) et implique la gestion de versions de documents. II est parfois fastidieux de parvenir à un ajustement mutuel dans la production d'un document satisfaisant, à la fois pour les ingénieurs pédagogiques et pour l'ensemble des enseignants impliqués.

\footnotetext{
${ }^{4}$ https://drive.google.com/drive/folders/10n2vbocYrh6Pid-o4Eoasn3yZvEUfnuP

${ }^{5}$ https://elearn.univ-pau.fr/course/index.php?categoryid $=6$
} 
- La démarche de scénarisation est actuellement formalisée par l'intermédiaire de modèles de documents qui offrent peu de flexibilité lorsqu'il devient nécessaire d'adapter certaines étapes / questions pour tenir compte des spécificités du module d'enseignement scénarisé ou encore du profil et/ou de la langue des enseignants impliqués dans le travail de scénarisation.

- La mise en ligne d'un nouveau module d'enseignement est fastidieuse et chronophage car, une fois le scénario pédagogique spécifié, elle est réalisée manuellement en reprenant et en synthétisant toutes les informations fournies dans le scénario.

Partant de ce constat, nous avons souhaité élaborer des modèles et une plateforme support :

- permettant à des ingénieurs pédagogiques de créer et adapter des démarches de scénarisation;

- facilitant le travail coopératif entre ingénieurs pédagogiques et enseignants;

- automatisant l'export d'un scénario pédagogique vers un LMS (Learning Management System) de type Moodle pour créer le cours correspondant.

Ces différents éléments de contribution sont présentés dans les sections suivantes.

\section{État de l'art}

De nombreuses définitions associées au terme scénario dans ses diverses déclinaisons et spécifications ont été proposées dans la littérature (Guéraud, 2005; Villiot-Leclercq, 2007; Bakki, 2018).

Comme défini par Paquette et Léonard (2013), un scénario est un ensemble ordonné d'activités pédagogiques (d'apprentissage ou d'assistance), régies par des acteurs qui utilisent et produisent des ressources ou "objets d'apprentissage " pouvant prendre plusieurs formes (texte, audio ou vidéo narrative). Le scénario d'apprentissage décrit le déroulement, et parfois les objectifs, les étapes, voire les consignes, les outils et documents utilisés ou à produire. Même si un scénario pédagogique est généralement conçu par un enseignant qui peut être assisté par un ingénieur pédagogique, d'autres acteurs (responsable, tuteur, etc.) peuvent aussi être impliqués dans son élaboration (Paquette, 2002) et (Basque, 2016).

Dans les travaux de (Guéraud, 2005), un scénario pédagogique vise trois objectifs : (1) définir en détail l'activité proposée aux apprenants; (2) spécifier de quelle manière la progression de l'apprentissage sera contrôlée; (3) décrire l'aide fournie tout au long de la progression de l'apprenant.

Comme le montrent les travaux cités précédemment, il existe plusieurs modèles extrêmement riches permettant de décrire les nombreuses facettes d'un scénario pédagogique. Dans le cadre de l'expérience menée pour le DU ASR, l'un des challenges était l'accompagnement de 18 enseignants pour élaborer, en 10 mois, 11 modules d'enseignement représentant un total de 500 heures de travail étudiant. Étant donné ces contraintes, il a été nécessaire de faire des choix en privilégiant les solutions simples et opérationnalisables dans le délai imparti. En ce sens, nous avons écarté les modèles de scénarios jugés trop riches et dont toutes les caractéristiques ne pouvaient pas être décrites lors des phases de spécification (par manque de temps) ou prises en compte de manière opérationnelle (pour raisons techniques) lors des phases de déploiement sur Moodle.

Par contre, nous avons considéré les travaux permettant de modéliser des éléments de scénarios importables sur un LMS :

- les standards permettant de modéliser des objets d'apprentissage via SCORM - Sharable Content Object Reference Model - (http://www.scorm.com/) ou IMS-LD - Instructional Management Systems Learning Design - (http://www.imsglobal.org/learningdesign). 
- les outils permettant de décrire la structure d'un scénario : SCENARI/OPALE (Crozat, 2011), LAMS (LAMS, 2014), CAPtuRe-platform (Chaabouni, 2017) ou G-MOT/TELOS (http://lice.licef.ca).

Vis-à-vis de nos objectifs, les outils de scénarisation nous ont permis d'imaginer les fonctionnalités nécessaires à une plateforme de scénarisation. Les standards offrent un cadre sur lequel s'appuyer pour traduire la spécification du scénario produit par les enseignants sous une forme normée importable sur un LMS.

$\mathrm{Au}$ cours de cette expérience, la collaboration socioconstructiviste (ingénieur pédagogique $\Leftrightarrow$ enseignants) mise en place par l'ingénieur pédagogique est comparable à la collaboration socioconstructiviste (enseignant $\Leftrightarrow$ étudiant) mise en place pour un enseignement par projet (Schneider et al., 2003). Selon cette approche, l'enseignant assure plusieurs rôles :

- celui de manager en veillant à la productivité des étudiants;

- celui de facilitateur pour assister les étudiants dans le choix des ressources utiles à la résolution des problèmes;

- celui d'orchestreur pour aider les étudiants à décomposer les problèmes en sous-problèmes.

En faisant le parallèle, l'ingénieur pédagogique a assuré, dans le cadre du DU ASR, les rôles de manager, de facilitateur et d'orchestreur pour assister les enseignants lors de l'élaboration des modules de la formation. L'ingénieur pédagogique a coordonné les enseignants en gardant une intention opérationnelle, l'objectif étant de mettre en ligne en 10 mois la totalité des modules de formation du DU ASR. Dans ce contexte, l'ingénieur pédagogique a abordé la tâche de scénarisation comme un travail de conceptualisation visant à :

- décrire le déroulement d'une situation d'apprentissage (Pernin et Lejeune, 2004),

- définir les objectifs, la planification des tâches, la description des tâches des apprenants et des modalités d'évaluation (Lando, 2003).

Compte tenu des courts délais de mise en œuvre de l'expérience conduite, les scénarios pédagogiques sur lesquels les enseignants ont travaillé n'intègrent pas formellement de notions pour une réingénierie ou une réutilisation desdits scénarios au sens de Barré et Choquet (2005) ou de Pernin et Godinet (2006).

Les modules du DU ASR devant être mis en ligne sur Moodle, l'ingénieur pédagogique a scénarisé les différents modules en se basant sur ses connaissances du modèle de scénario sous-jacent à Moodle (Loiseau et al., 2017).

Ce modèle (Figure 2) permet aux enseignants concepteurs d'opérationnaliser un scénario en créant des cours composés de sections dans lesquelles les apprenants trouveront des ressources (fichiers, dossiers...) et des outils (référencés sous le terme ambigu d'activités) tels que des questionnaires à choix multiple, des forums, des wikis, etc. 


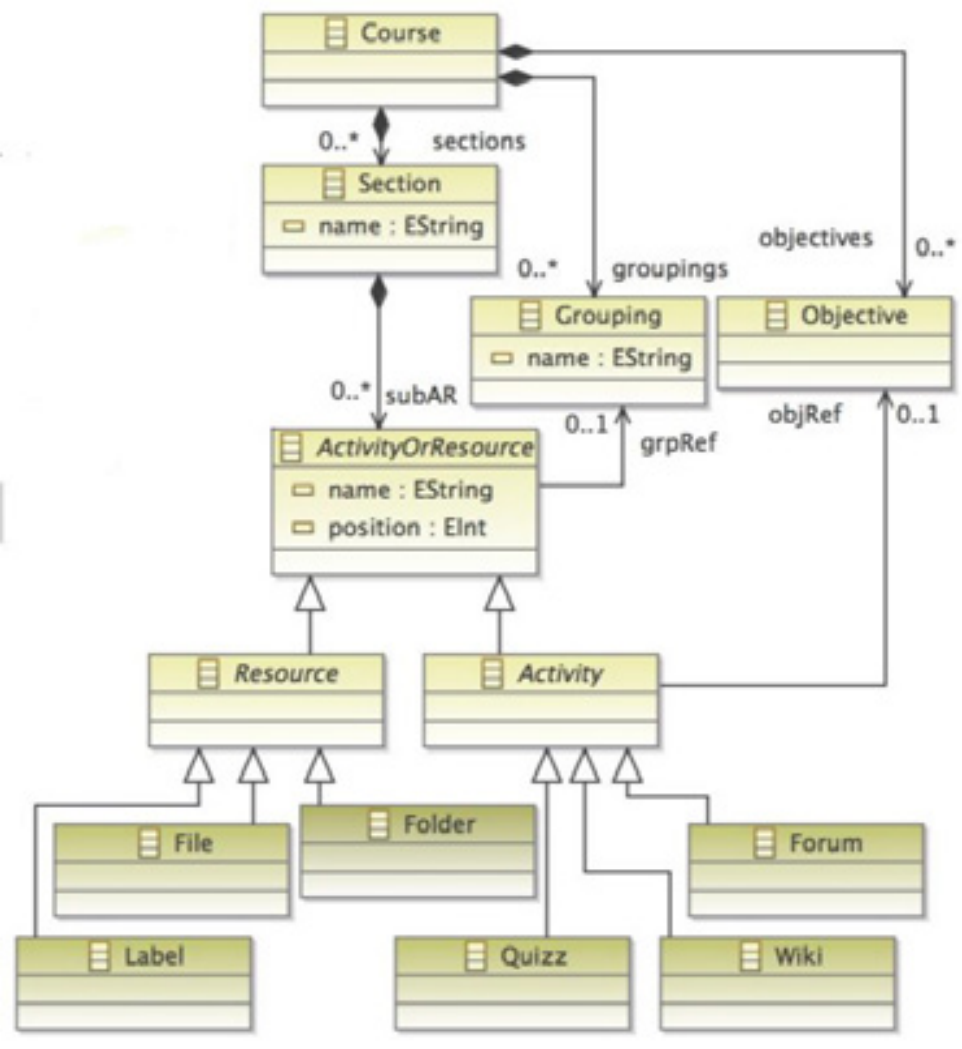

Figure 2. Modèle de scénario intégré dans Moodle (Loiseau et al., 2017)

D'un point de vue méthodologique, l'ingénieur pédagogique a élaboré les modules de formation du DU ASR selon cinq phases, conformément au modèle ADDIE - Analysis, Design, Development, Implementation and Evaluation - (Branch, 2009). Ce modèle (Figure 3) est à la base de nombreuses méthodes de design pédagogique (Basque, 2004) car il identifie les phases du cycle de vie d'un système d'apprentissage selon une approche classique des méthodes de conception logicielle : Analyse, Conception, Développement, Implantation, Évaluation (Kurt, 2019).

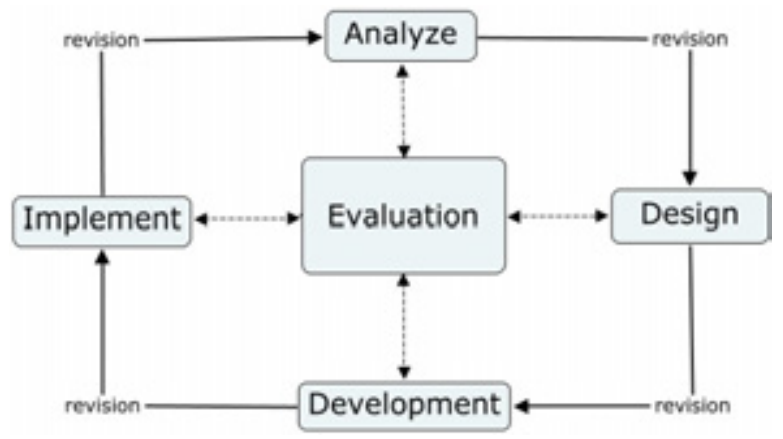

Figure 3. Modèle ADDIE (Kurt, 2017)

Ce modèle a été préféré au modèle SAM - Successive Approximation Model - (Allen et Sites, 2012), dont la démarche itérative associée relève de l'approche "agile » (Figure 4). L'agilité vise à satisfaire les besoins du client, non pas en suivant un cadre strict prédéfini et considéré comme immuable mais sur la 
base d'une construction itérative, incrémentale et adaptative du produit final. Elle repose également sur la collaboration avec les clients qui doit être un partenaire proactif dès les premières phases et tout au long du cycle de vie. Dans ce cadre, SAM repose sur trois étapes : Évaluation, Conception et Développement, avec un ensemble d'itérations sur l'ensemble du processus, qui peut donner lieu à la remise en cause du résultat obtenu et une reprise, à chaque jalon, de l'étape d'Évaluation.

Malgré les avantages du modèle SAM, les modules du DU ASR ont été conçus selon le modèle ADDIE qui offre un caractère plus structurant: chaque étape peut faire l'objet d'itérations en vue de son perfectionnement et se termine nécessairement par une validation avant de passer à l'étape suivante.

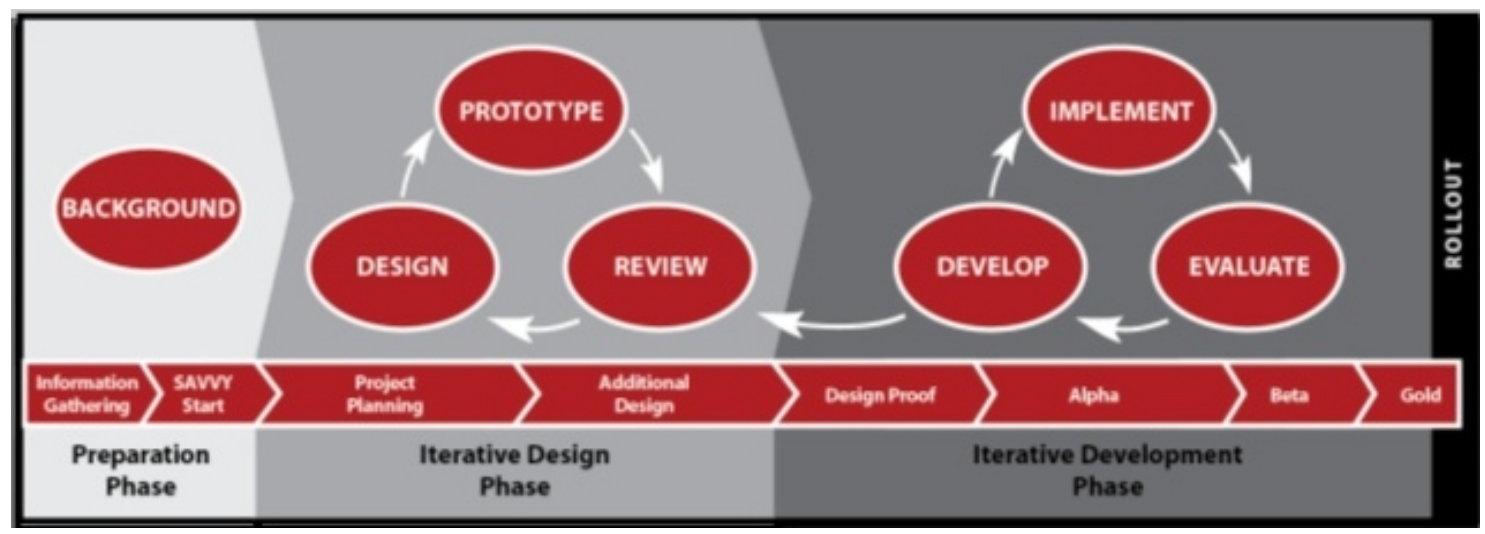

Figure 4. Modèle SAM (Allen et Sites, 2012)

En nous basant sur l'expérience acquise sur le DU ASR, notre objectif est de concevoir des modèles permettant d'élaborer des scénarios pédagogiques en garantissant l'opérationnalisation de ces scénarios sur Moodle. Nous pouvons noter deux approches pour traiter ce problème :

- les travaux proposant des outils ad hoc de spécification de scénarios générant une spécification qu'il faut ensuite mettre en correspondance avec les données attendues par Moodle (Alario-Hoyos et al., 2012; Katsamani et al., 2012). De par la dissimilarité importante entre le langage de scénarisation et le modèle de données du LMS, ces approches induisent souvent des modifications et des pertes sémantiques lors du processus de mise en correspondance des données du scénario spécifié avec les données attendues par le LMS.

- les travaux basés sur l'Ingénierie Dirigée par les Modèles (IDM) pour transformer un scénario spécifié avec un modèle donné vers le modèle de Moodle (Abdallah et al., 2008). Toutefois, ces transformations se révèlent complexes à modéliser et induisent souvent une intervention manuelle et des pertes sémantiques.

Pour éviter ces pertes sémantiques, nous avons fait le choix, à l'instar de Loiseau et al. (2017), de partir directement du modèle de scénario implanté dans le LMS Moodle et de proposer aux ingénieurs pédagogiques des facilités pour concevoir des démarches permettant de scénariser conformément à ce modèle. Nous souhaitons que les démarches proposées puissent être à la fois rigoureuses et structurantes comme ADDIE, mais suffisamment souples comme SAM pour permettre à l'ingénieur pédagogique d'adapter/affiner sa manière d'accompagner les enseignants concepteurs. À cette fin, nous proposons un modèle pour construire des démarches de scénarisation en tenant compte du modèle de scénario attendu par le LMS. 


\section{Modèles pour la scénarisation pédagogique}

\section{Modèle de scénario}

La définition des éléments composant un scénario pédagogique a été menée en collaboration avec l'ingénieur pédagogique. Nous avons effectué un travail d'analyse pour nous imprégner de son vocabulaire afin de produire un modèle de scénario correspondant à sa vision (Figure 5). Le vocabulaire employé par l'ingénieur pédagogique dans la démarche de spécification des modules du DU ASR était issu de son expérience et de sa connaissance de la plateforme Moodle sur laquelle le scénario allait être déployé : un scénario pédagogique (ou module de cours) est composé d'un ensemble de chapitres (ou séquences pédagogiques). Chaque chapitre se décompose en séances dans lesquelles les apprenants utilisent des ressources (fichier PDF, vidéo, QCM, forum, chat...) pour mener une activité donnée.

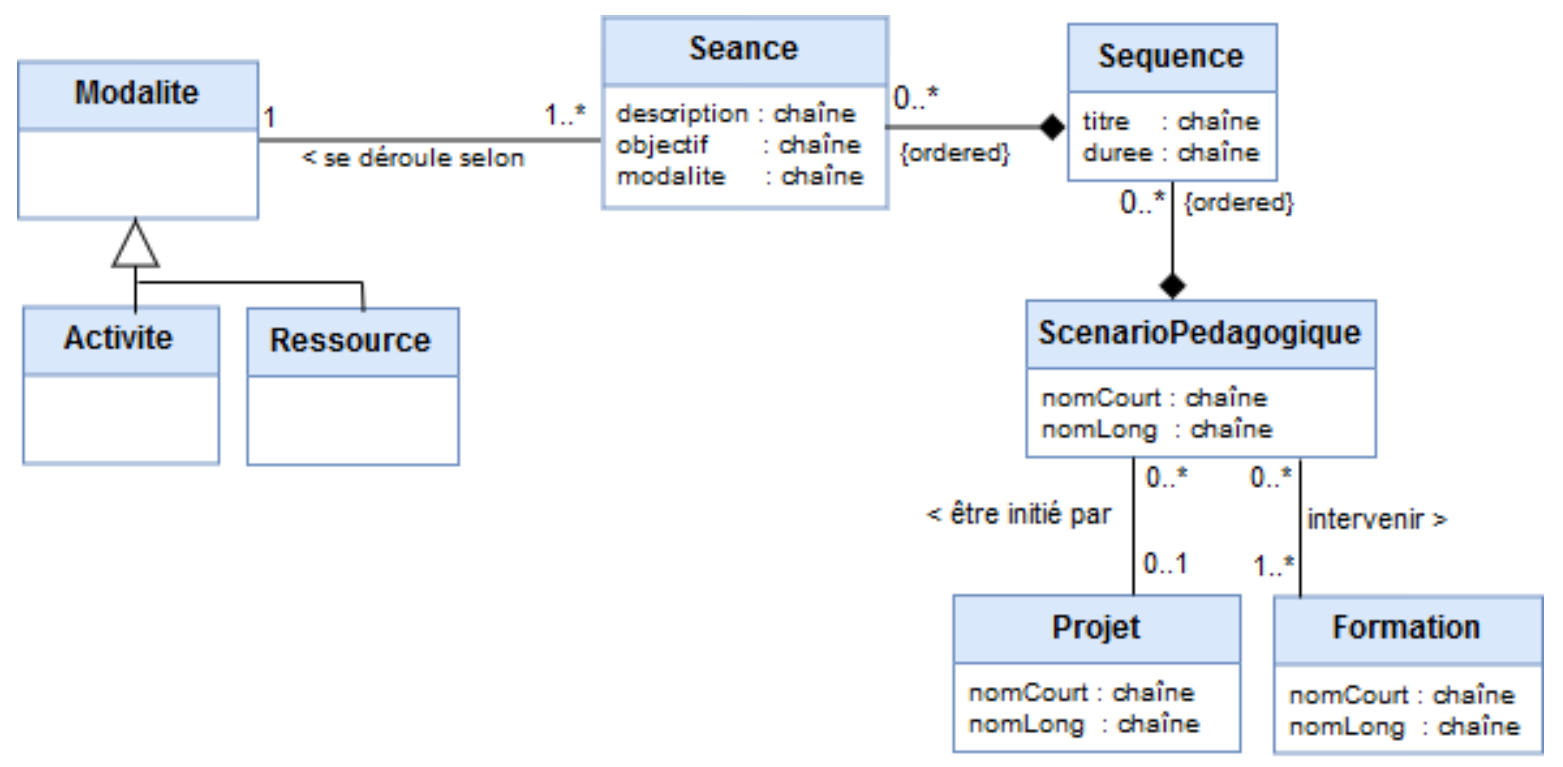

Figure 5. Modèle de scénario basé sur la vue de l'ingénieur pédagogique

Nous avons tout de même fait un lien entre le vocabulaire manipulé par l'ingénieur pédagogique et les concepts propres à la plateforme Moodle, de sorte à être en mesure d'exporter sur Moodle tous les éléments de scénarios définis par l'ingénieur avec les enseignants (Tableau 1).

Tableau 1. Correspondance entre les concepts pédagogiques manipulés et les concepts Moodle

\begin{tabular}{|c|c|}
\hline Vocabulaire de l'ingénieur pédagogique & Concepts Moodle associés \\
\hline Module - Scénario pédagogique & Cours \\
\hline Chapitre - Séquence pédagogique & Section \\
\hline Séance & Activité - Ressource \\
\hline
\end{tabular}




\section{Modèle de démarche de scénarisation}

Au-delà de la modélisation des éléments constituant un scénario, nous avons souhaité capitaliser l'expérience menée lors de la mise en place des modules du DU ASR. À cette fin, nous avons dans un premier temps formalisé la démarche de scénarisation employée de sorte à faciliter sa réutilisation. Dans un second temps, nous avons généralisé le concept de démarche de scénarisation pour permettre aux ingénieurs pédagogiques d'adapter la démarche utilisée dans le cadre du DU ASR ou bien pour élaborer de nouvelles démarches.

Selon notre approche, une démarche de scénarisation est conçue par un ingénieur pédagogique. La mise en œuvre d'une démarche est menée de manière coopérative entre un ou plusieurs enseignants et un ou plusieurs ingénieurs pédagogiques qui accompagnent ces enseignants dans ce travail de spécification du scénario. L'expérience menée dans le cadre de la définition des modules du DU ASR a montré qu'il était important de désigner un enseignant responsable lorsque plusieurs enseignants participent à la scénarisation d'un même module. Cet enseignant responsable est l'interlocuteur privilégié de l'ingénieur pédagogique lorsque celui-ci doit coordonner le travail de spécification de l'équipe enseignante.

Dans la suite de cette section, le terme ingénieur pédagogique pourra aussi bien désigner un ingénieur seul ou plusieurs ingénieurs coordonnant le travail de spécification. De même, le terme enseignant pourra désigner l'enseignant unique qui réalise le travail de spécification avec l'ingénieur pédagogique ou bien l'enseignant responsable qui coordonne le travail de spécification réalisé par lui-même et ses collègues.

La figure 6 décrit le modèle que nous avons élaboré à cette fin.

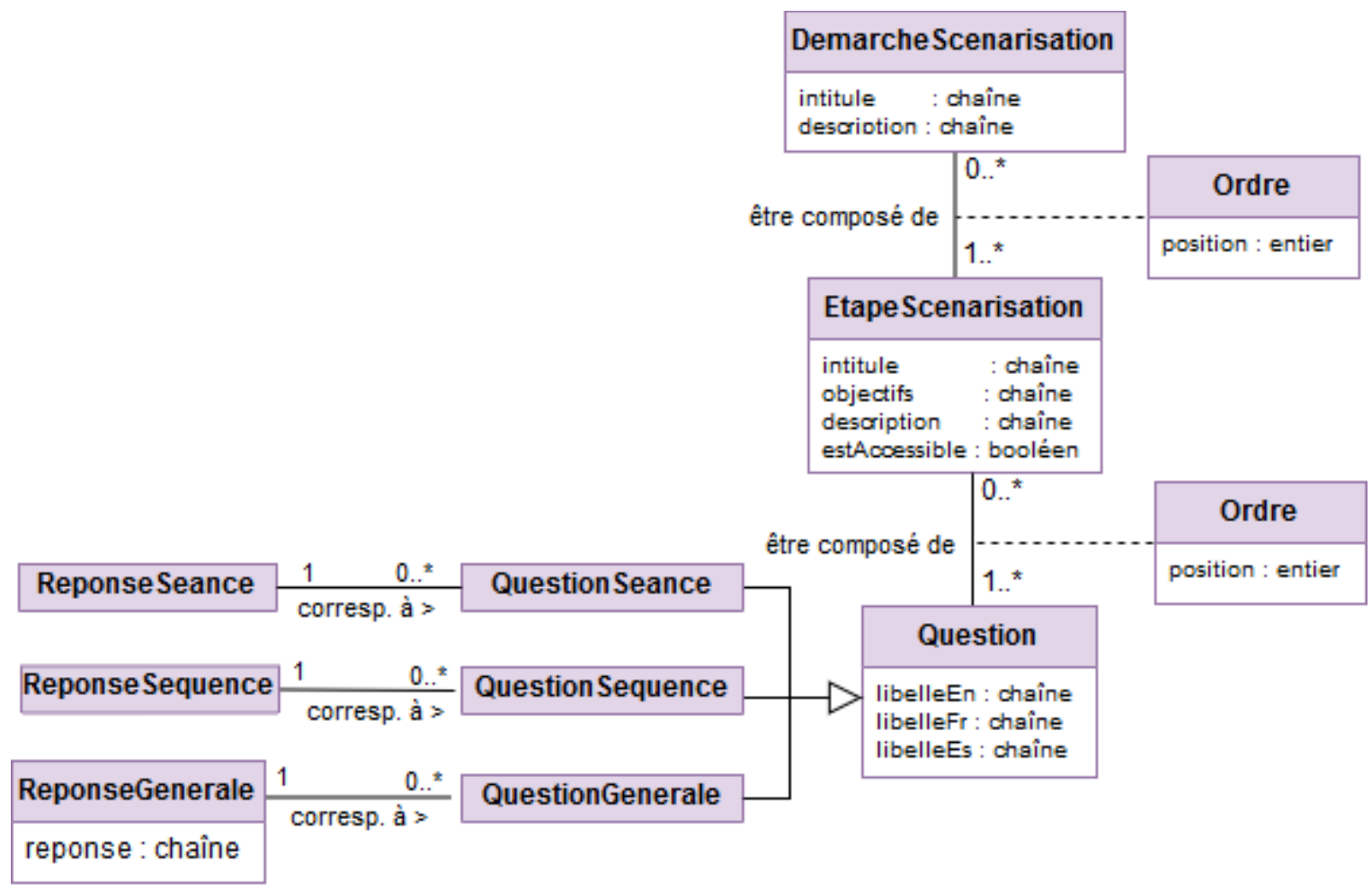

Figure 6. Modèle support pour l'élaboration de nouvelles démarches de scénarisation 
Dans le cadre de notre étude, et comme modélisé dans la partie en haut à droite de la figure 6 , une démarche de scénarisation (classe DemarcheScenarisation) est composée d'un ensemble d'étapes (classe EtapeScenarisation) ordonnées (classe Ordre et propriété position) ayant chacune pour objectif de spécifier une partie du scénario.

Les objectifs (propriété objectifs de la classe EtapeScenarisation) de spécification de chaque étape sont définis par l'ingénieur pédagogique. C'est aussi l'ingénieur pédagogique qui définit, selon le degré d'avancement du travail de scénarisation, si l'étape suivante est actuellement accessible ou pas à l'enseignant (propriété estAccessible).

Comme suggéré dans ADDIE, cette propriété permet à l'ingénieur pédagogique de contrôler la progression du travail de spécification en donnant accès à une nouvelle étape de scénarisation (classe EtapeScenarisation) uniquement si le travail de spécification de l'étape précédente est jugé satisfaisant et terminé.

Une étape de scénarisation est composée d'un ensemble de questions (classe Question) dont le contenu et l'ordre sont définis par l'ingénieur. Ces questions seront posées à l'enseignant afin que celui-ci spécifie les propriétés de son scénario pédagogique. Puisque nous avons considéré qu'un scénario était composé de séquences, elles-mêmes composées de séances (Figure 5), les questions définies par l'ingénieur pédagogique devront amener l'enseignant à spécifier ces éléments. Le modèle distingue trois types de questions (partie en bas à gauche de la figure 6) :

- les questions générales (classe QuestionGenerale) n'ont pas de lien direct avec le scénario à élaborer. II peut s'agir de questions qui permettent à l'ingénieur pédagogique de mieux cerner l'enseignant avec qui il coopère (compétences en scénarisation, niveau de connaissance sur les TICE...) ou bien des questions qui vont préparer l'enseignant à l'identification des séquences et séances de son scénario;

- les questions de type séquence (classe QuestionSequence) visent à spécifier précisément les séquences composant le scénario élaboré;

- les questions de type séance (classe QuestionSeance) spécifient les séances de chaque séquence.

Les réponses apportées (classes ReponseGenerale, ReponseSequence et ReponseSeance sur les figures 6 et 7) correspondent à la spécification des séquences et des séances (classes Sequence et Seance sur la figure 7) du scénario à déployer ultérieurement (classe ScenarioPedagogique) sur la plateforme pédagogique.

Cette figure 7 ne détaille pas la partie de gestion des utilisateurs (classes Enseignant et IngenieurPedagogique), mais le modèle intègre des propriétés permettant d'identifier un enseignant référent pour chaque scénario. Cet enseignant est chargé de coordonner les propositions de ses collègues lorsque le module est conçu par plusieurs enseignants et c'est lui qui est en relation directe avec l'ingénieur pédagogique. 


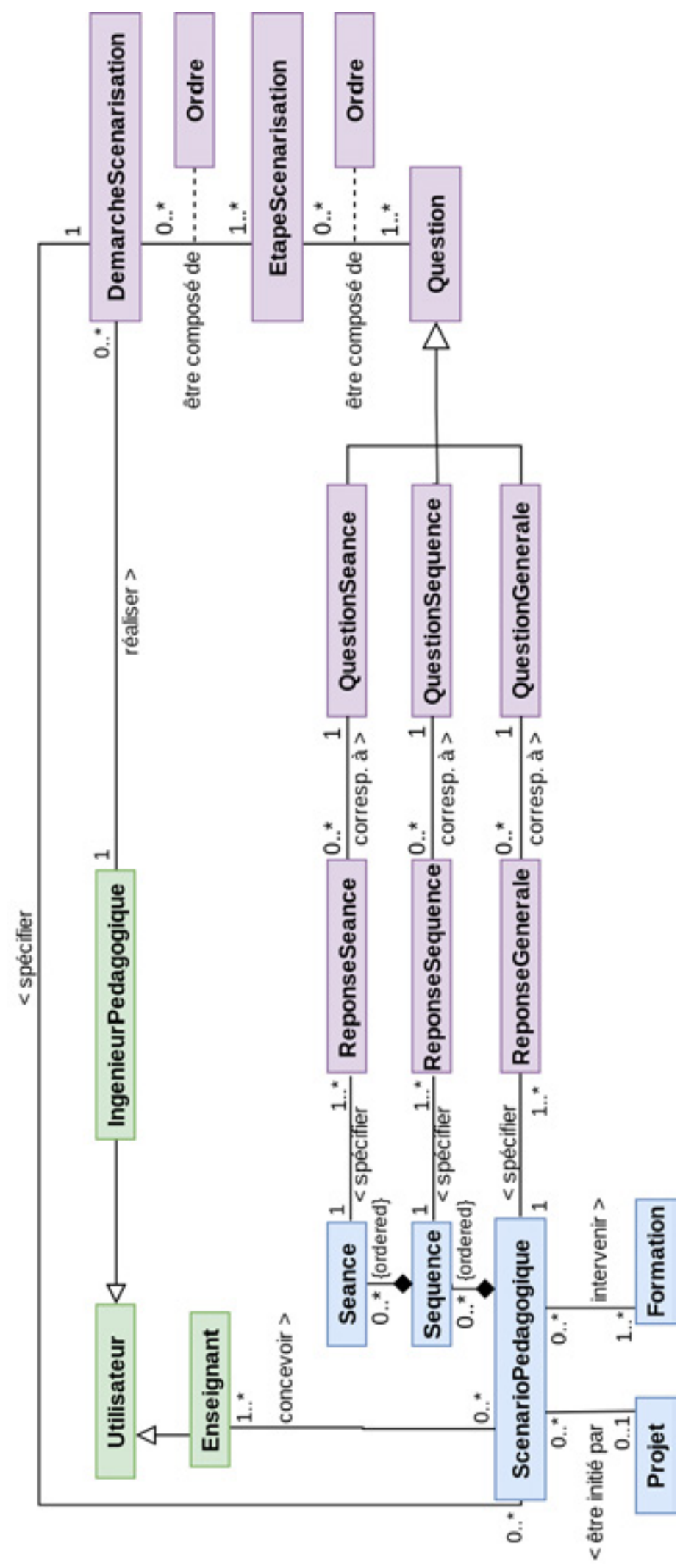

Figure 7. Modèle général décrivant le rôle des acteurs impliqués dans une démarche de scénarisation visant à définir les séquences et les séances d'un scénario pédagogique

revue-mediations.teluq.ca | No. 2, 2019 


\section{Une plateforme support à la conception et à la mise en œuvre de démarches de scénarisation}

Pour évaluer l'intérêt et la pertinence de notre approche, nous avons intégré nos modèles dans une plateforme de scénarisation coopérative afin de mesurer leur pertinence lors de la conception de nouveaux modules. Cette plateforme permet à des ingénieurs pédagogiques de concevoir des démarches de scénarisation puis de les mettre en œuvre afin de scénariser des cours en coopération avec des enseignants.

La conception de démarches de scénarisation se veut flexible et peut être menée de manière :

- descendante : définition des objectifs de la démarche, élaboration des étapes composant la démarche puis spécification des questions à poser aux enseignants à chaque étape;

- ascendante : élaboration d'un ensemble de questions pertinentes pour spécifier un scénario puis répartition de ces différentes questions au sein d'étapes;

- mixte : création par exemple d'une première étape puis définition des questions associées, création ensuite d'une deuxième étape puis définition des questions associées, etc.

Pour soutenir cette approche de conception flexible, la plateforme de scénarisation propose aux ingénieurs pédagogiques d'élaborer dans l'ordre qu'ils souhaitent :

- des questions qui seront ajoutées à une banque de questions et qui pourront ultérieurement être ajoutées à des étapes (Figure 8);

- des étapes qui seront ajoutées à une banque d'étapes et qui pourront ultérieurement être utilisées pour former une démarche spécifique.

Scénarisation / Banque de questions

2 Gaizka Alçuyet c- Se déconnecter

\section{Liste des questions}

Libellé français

Actions

Comment vous appelez vous?

Comment estimez vous votre aisance avec les outils numériques ?

Combien de cours avez vous conç par le passé ?

\section{+ Ajouter une question}

Figure 8. Interface d'édition de la banque de questions qui serviront à l'ingénieur pédagogique pour structurer les étapes d'une démarche de scénarisation

Si la démarche de scénarisation est construite de manière descendante, l'ingénieur pédagogique :

1) nommera la démarche qu'il/elle souhaite créer; 
2) intègrera dans cette démarche les étapes qui la composent en piochant dans la banque d'étapes (Figure 9) ou en créant à la volée des étapes (dans ce dernier cas, la conception de la démarche est mixte);
1 Gaizka Alçuyet C- Se déconnecter

\section{Association d'étapes de scénarisation}

Dimarche courante : Dimarche de Gaizka

Etapes de scénarisation associées

\begin{tabular}{|l|l|l|l|} 
& Position & Intitulé & Actions \\
\hline$\Psi$ & 1 & Brise glace & S \\
\hline$\Psi$ & 2 & Définir les séquences & S \\
\hline & 3 & Définir les séances & S \\
\hline
\end{tabular}

scenarisationProcess.otherStages:

Intitulé

Lister les difficultés

Actions

8

Figure 9. Interface de création d'une démarche de scénarisation à partir d'étapes disponibles dans la banque d'étapes

3) définira pour chaque étape les questions à poser en piochant dans la banque de questions (Figure 10) ou en créant à la volée des questions (dans ce dernier cas, la conception de la démarche est mixte).

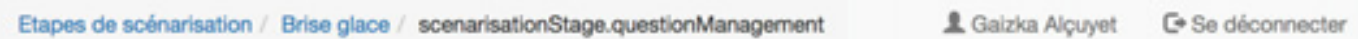

\begin{tabular}{|l|l|l|l|}
\hline & Position & Libellé français & Actions \\
\hline 4 & 1 & Combien de cours avez vous conçu par le passé ? & $\$ 3$ \\
\hline & 2 & Comment estimez vous votre aisance avec les outils numériques ? & $\$$ \\
\hline
\end{tabular}

Autres questions disponibles :

Libellé français

Actions

A combien estimez vous le nombre de sections de votre cours?

Figure 10. Interface permettant de définir les questions posées à l'enseignant lors d'une étape de scénarisation 
Si la démarche de scénarisation est construite de manière ascendante, l'ingénieur pédagogique :

1) définira les questions qu'il/elle envisage d'utiliser dans sa démarche;

2) créera (nommera) les étapes qui composeront sa démarche;

3) associera les questions à chacune des étapes créées (Figure 10) ;

4) assemblera et ordonnera les étapes précédemment créées pour finaliser sa démarche (Figure 9).

Cette approche de conception flexible nous a permis de modéliser et mettre en ligne la démarche de scénarisation employée pour élaborer les modules d'enseignement du DU ASR (cf. Section 2). Cette démarche en ligne est actuellement utilisée pour élaborer de nouveaux modules d'enseignement dans une autre formation dédiée aux TIC (https://bit.ly/2HIVBWd) ${ }^{6}$.

\section{Discussion relative à la scénarisation coopérative}

L'expérience menée pour le DU ASR a mis en évidence les nombreux échanges nécessaires entre l'ingénieur pédagogique et l'enseignant pour spécifier un scénario pédagogique. Ces échanges prenant la forme de mails et de visioconférences ne facilitaient pas le suivi du travail de spécification: relance régulière des enseignants, demande de précision sur des questions posées par l'ingénieur pédagogique, demande de précision sur des réponses données par l'enseignant, etc.

La version courante de la plateforme basée sur les modèles présentés permet de spécifier les séquences et les séances d'un scénario mais ne trace pas les différents échanges conduisant au scénario final et ne propose pas vraiment un support pour faciliter les échanges et la coopération entre les différents acteurs. Dans cette section, nous abordons, sous forme de discussion, quelques idées pour répondre à ce manque.

Les problèmes que nous pouvons soulever sont les suivants :

- Comment faciliter le travail de l'ingénieur pédagogique pour coordonner l'avancement du travail de spécification?

- Comment permettre à l'enseignant de suivre cette progression?

- Comment accroître le sentiment d'implication des enseignants tout au long du processus de conception?

Pour répondre à ces points, nous imaginons élaborer un dispositif qui soit à la fois partagé par les enseignants et l'ingénieur pédagogique et qui supporte une coordination basée à la fois sur de la supervision directe et de l'ajustement mutuel. La supervision permettra à l'ingénieur pédagogique de rythmer la progression du travail en décidant quand passer à l'étape de spécification suivante. L'ajustement mutuel qui privilégie les échanges entre les acteurs concernés permettra d'impliquer les enseignants dans le processus de conception. C'est également le mode de coordination privilégié pour coordonner l'action de plusieurs personnes réparties dans plusieurs services ou plusieurs unités géographiques (Mintzberg et al., 2005).

Pour mettre en place une coordination basée à la fois sur de la supervision directe et de l'ajustement mutuel, nous envisageons d'élaborer un tableau de bord consignant les différents échanges de la coopération entre enseignants et ingénieur pédagogique. Le contenu de ce tableau de bord pourra reprendre les étapes de la démarche de scénarisation choisie par l'ingénieur pédagogique et indiquer pour chaque étape le taux d'avancement ainsi que l'acteur chargé de réaliser la prochaine tâche. Le fonctionnement général de ce dispositif de coopération est décrit et maquetté dans les figures suivantes.

\footnotetext{
${ }^{6}$ https://www.iutbayonne.univ-pau.fr/espace-entreprises/modules-formation.html
} 
Initialement l'ingénieur pédagogique choisit une démarche de scénarisation et rend accessible la première étape (propriété estAccessible de la classe EtapeScenarisation de la Figure 6). II sollicite ensuite l'enseignant référent pour répondre aux questions de cette première étape (Figure 11).

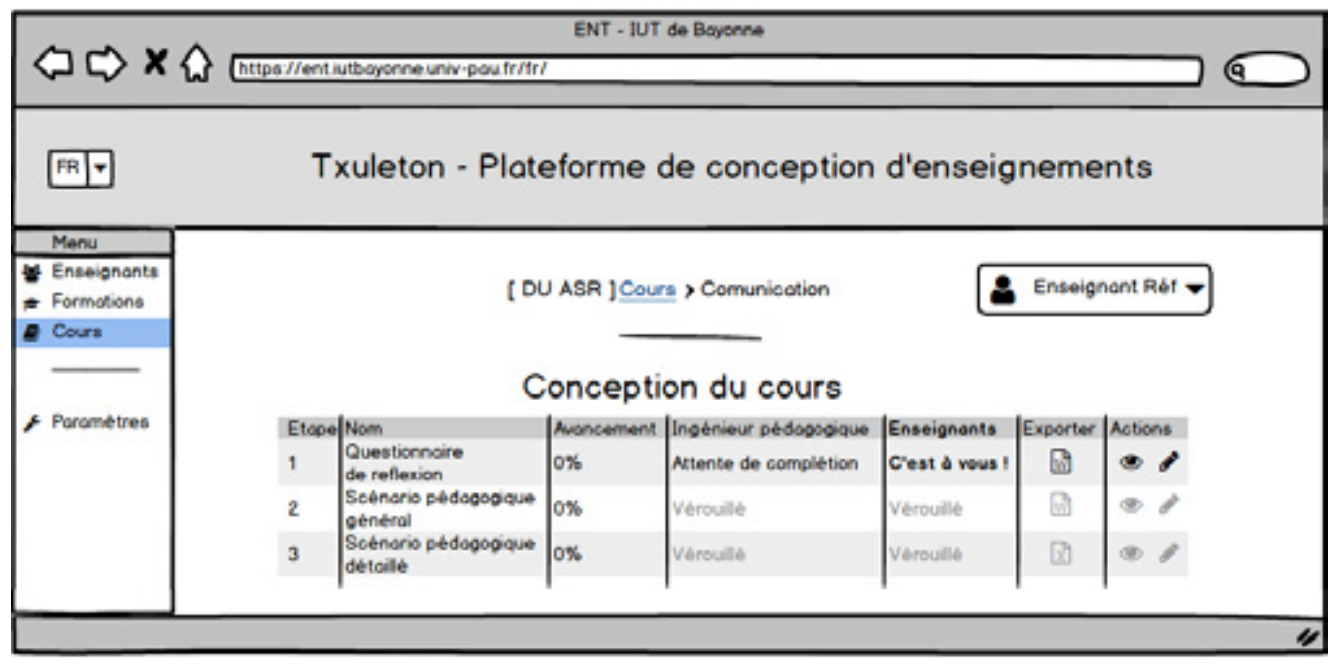

Figure 11. Sollicitation de l'enseignant pour répondre aux questions de l'étape 1

L'enseignant accède ensuite aux questions de la première étape, y répond puis notifie l'ingénieur pédagogique (Figure 12).

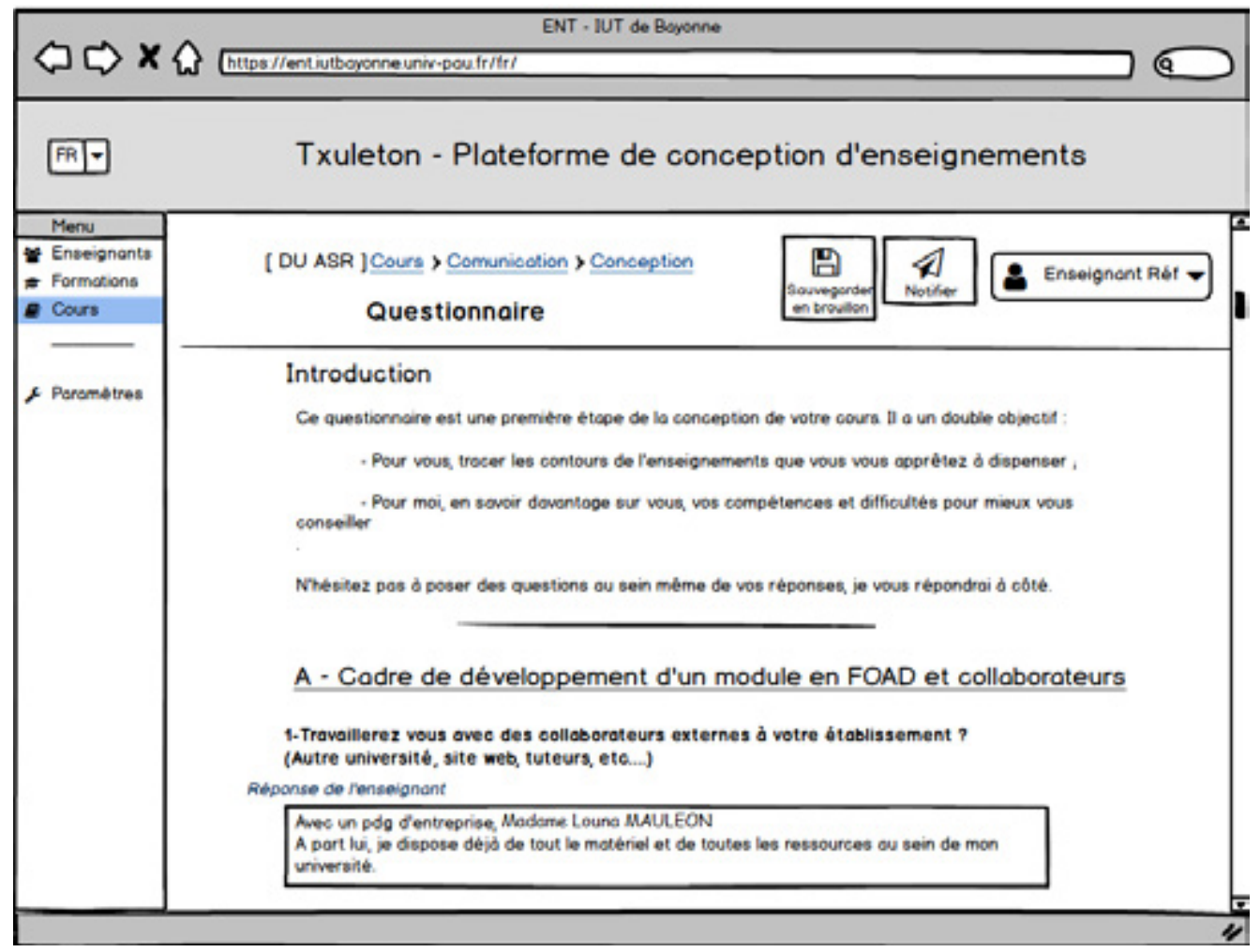

Figure 12. Réponses de l'enseignant aux questions définies pour l'étape courante 
L'ingénieur pédagogique est notifié des réponses de l'enseignant et doit les valider (Figure 13).

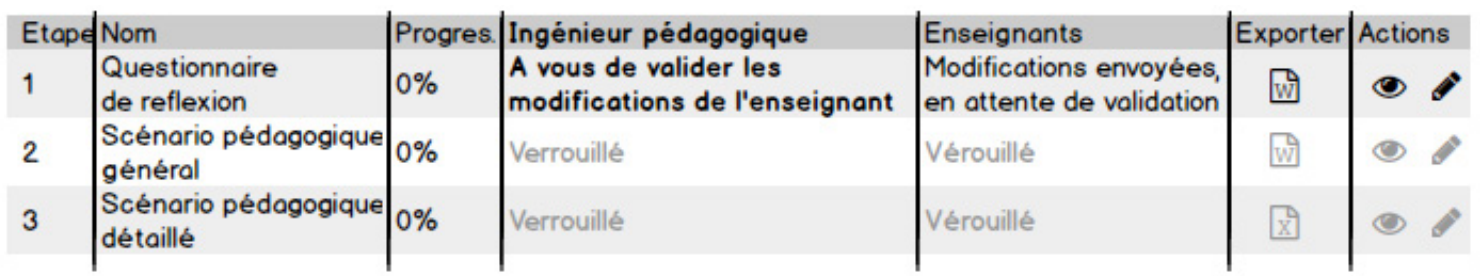

Figure 13. Validation en attente des réponses fournies par l'enseignant pour l'étape courante de la démarche

L'ingénieur pédagogique valide les réponses jugées complètes; si nécessaire il sollicite de nouveau l'enseignant pour des compléments d'informations sur les réponses incomplètes ou imprécises (Figure 14). En fonction du nombre de questions de l'étape et du nombre de réponses validées par l'ingénieur pédagogique, un taux de progression est affiché dans le tableau de bord pour l'étape en cours (ici 50\%). Le mécanisme de validation des réponses permet à l'ingénieur pédagogique de contrôler la progression de la spécification au sein d'une étape.

\begin{tabular}{|c|c|c|c|c|c|c|}
\hline Etape & Nom & Progres & Ingénieur pédagogique & Enseignants & Exporter & Actions \\
\hline 1 & $\begin{array}{l}\text { Questionnaire } \\
\text { de reflexion }\end{array}$ & $50 \%$ & $\begin{array}{l}\text { Remarques envoyées, er } \\
\text { attente de modification }\end{array}$ & $\begin{array}{l}\text { Enseignant ; Remarques reçues } \\
\text { modifications d̀ apporter }\end{array}$ & w & (1) \\
\hline 2 & $\begin{array}{l}\text { Scénario pédagogiqu } \\
\text { général }\end{array}$ & $0 \%$ & Verrouillé & Vérouillé & w & 0 \\
\hline 3 & $\begin{array}{l}\text { Scénario pédagogiqu } \\
\text { détaillé }\end{array}$ & $0 \%$ & Verrouillé & Vérouillé & X & 0 \\
\hline
\end{tabular}

Figure 14. Validation partielle des réponses fournies par l'enseignant pour l'étape courante de la démarche

Si une étape est remplie à 100\%, la plateforme débloque automatiquement l'étape suivante (Figure 15). Si une étape n'est pas totalement renseignée, l'ingénieur pédagogique peut quand même choisir de débloquer l'étape suivante.

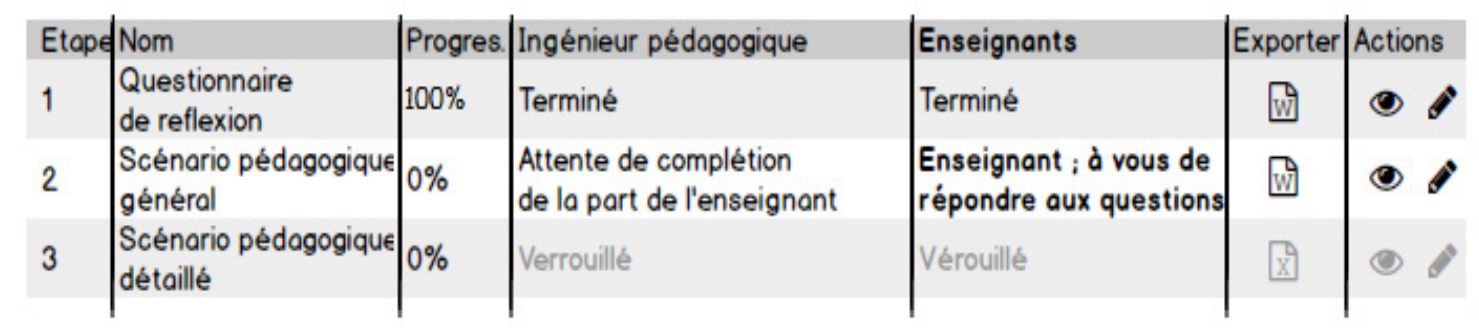

Figure 15. Validation de l'étape courante par l'ingénieur pédagogique et initialisation de l'étape suivante

A tout moment, l'enseignant et l'ingénieur pédagogique ont la possibilité d'exporter leur travail en cours sous forme de document imprimable. Cette fonctionnalité est mise à disposition pour rassurer les enseignants peu habitués à la FOAD ou qui collaborent pour la première fois avec l'ingénieur pédagogique.

Une coordination basée sur ce principe d'ajustement mutuel nous semble bénéfique pour l'ensemble des acteurs qui se sentent davantage impliqués dans la conception du scénario. Nous espérons que la mise 
en place d'un tel environnement coopératif facilitera d'une part le travail de l'ingénieur pédagogique pour coordonner l'avancement du travail de spécification et permettra d'autre part à chaque enseignant de constater la construction progressive de son module d'enseignement et donc de se sentir plus impliqué.

\section{Conclusion}

Les modèles de conception implantés au sein de la plateforme de scénarisation sont actuellement en phase d'évaluation. L'évaluation porte sur la pertinence (mais aussi les limites) des modèles permettant de concevoir de nouvelles démarches de scénarisation ou adapter des démarches existantes.

Le modèle de scénarios et le modèle de démarches de scénarisation représentent une première étape permettant à des ingénieurs et des enseignants d'élaborer la trame d'un scénario (séances et séquences) et de l'exporter sur le LMS Moodle. Les modèles proposés ne permettent actuellement pas de préciser / élaborer les ressources pédagogiques impliquées dans chaque séquence d'apprentissage. Ces ressources sont donc actuellement définies directement sur Moodle et non au niveau de la plateforme de scénarisation. Les prochains travaux visent donc à étendre le modèle de scénario pour supporter la spécification des ressources pédagogiques impliquées dans chaque activité d'apprentissage. L'extension du modèle de scénario suppose également l'extension du modèle de démarches de scénarisation pour pouvoir aussi spécifier cette dimension.

Une autre perspective de travail concerne les questions soulevées dans la section précédente autour de la prise en compte des aspects coopératifs dans la scénarisation. II s'agit d'étendre le modèle et la plateforme de conception non seulement pour intégrer le dispositif de coopération décrit mais aussi pour mesurer s'il permet effectivement de faciliter le travail de coordination de l'ingénieur pédagogique tout en améliorant l'implication des enseignants face à une coopération distante et asynchrone.

Afin de couvrir une plage de pratiques pédagogiques plus large et de rationaliser la progression des activités à conduire pour créer les modules d'enseignement, le modèle général doit être étendu pour prendre en considération les rôles pédagogiques communément identifiés dans l'état de l'art. Citons par exemple les rôles identifiés par Paquette (2002) et Basque (2016) responsable du module, auteur du module, scénariste, médiatiseur, tuteur, etc.

De manière plus large, il convient de cerner plus précisément le type de démarches de scénarisation qui peuvent être conçues avec nos modèles pour identifier les pratiques pédagogiques qui ne sont pas couvertes et pour faire évoluer nos modèles en conséquence.

\section{Liste de références}

Abdallah, F., Toffolon, C. \& Warin, B. (2008). Models Transformation to Implement a Project-Based Collaborative Learning (PBCL) Scenario: Moodle Case Study. Dans Eighth IEEE International Conference on Advanced Learning Technologies, p. 639-643.

Alario-Hoyos, C., Muñoz-Cristóbal, J., Prieto, L., Bote-Lorenzo, M., Asensio-Pérez, J., Gómez-Sánchez, E., Vega-Gorgojo, G. \& Dimitriadis, Y. (2012). GLUE! - GLUE!-PS: An approach to deploy non-trivial collaborative learning situations that require the integration of external tools. Dans VLEs. In First Moodle Research Conference, p. 77-85.

Allen, M. \& Sites, R. (2012). Leaving ADDIE for SAM: An Agile Model for Developing the Best Learning Experiences. American Society for Training \& Development,

Bakki, A. (2018). Modèle et outil pour soutenir la scénarisation pédagogique de MOOC connectivistes (Mémoire de thèse de doctorat, Université du Maine, Le Mans; Université Ibn Zohr, Agadir). Repéré à https://tel.archives-ouvertes.fr/tel-02021067 
Barré, V. \& Choquet, CH. (2005). Une aide à la réingénierie d'un scénario pédagogique via la préconisation et la formalisation d'observables. Dans Actes de la conférence EIAH 2005.

Basque, J. (2004). En quoi les TIC changent-elles les pratiques d'ingénierie pédagogique du professeur d'université? Revue Internationale des Technologies en Pédagogie Universitaire, 1(3), 7-13.

Basque, J. (2016, Oct.). Mener un projet d'approche-programme en enseignement supérieur : une démarche d'ingénierie pédagogique. Communication sur invitation présentée (à distance) à la formation « Approche-programme et approche par compétences dans l'enseignement supérieur » - Institut Français de l'Éducation.

Branch, R. M. (2009). Instructional Design: The ADDIE Approach. Springer-Verlag US, 203 pages. https://doi.org/10.1007/978-0-387-09506-6

Chaabouni, M. (2017). Assistance à la réutilisation de scénarios d'apprentissage : une approche guidée par l'évaluation du contexte d'usage à base d'indicateurs (Mémoire de thèse de doctorat, Université du Maine, Le Mans). Repéré à https://tel.archives-ouvertes.fr/tel-01808541

Charlier, B., Deschryver, N. \& Peraya, D. (2006). Apprendre en présence et à distance : Une définition des dispositifs hybrides. Distances et savoirs, 4(4), 469-496. https://www.cairn.info/revue-distances-etsavoirs-2006-4-page-469.htm

Crozat, S. (2011). Scenari - La chaîne éditoriale libre : Structurer et publier textes, images et son. Accès libre. Eyrolles.

Guéraud, V. (2005). Approche auteur pour les Situations Actives d'Apprentissage : Scénarios, Suivi et Ingénierie (Habilitation à Diriger les Recherches, Université Joseph Fourier, Grenoble). Repéré à https://telearn.archives-ouvertes.fr/hal-00190220/document

Katsamani, M., Retalis, S. \& Boloudakis, M. (2012). Designing a Moodle course with the CADMOS learning design tool. Educational Media International, 49(4), 317-331.

Kurt, S. (2017). ADDIE Model: Instructional Design, in Educational Technology. Repéré à https://educationaltechnology.net/the-addie-model-instructional-design

Kurt, S. (2019). An Introduction to the ADDIE Model: Instructional Design: The ADDIE Approach. Independently published (February 2, 2019), 59 pages, ISBN-10: 1723982423.

LAMS. (2014). Proceedings of the 9th International LAMS and Learning Design Conference, Nanyang Technological University (2014). Singapore. LAMS Foundation.

Lando, P. (2003). Progetto : une méthode de conception de gabarits de scénarios pour activités pédagogiques collectives distantes à base de projets. (Mémoire de DEA, Université de Picardie, France).

Loiseau, E., Laforcade, P., Mawas, N.E. \& Iksal, S. (2017). Abstraction des fonctionnalités d'une plateforme de formation pour la mise en œuvre de langages de scénarisation. Revue STICEF, 24(1).

Mintzberg, H., Ahlstrand, B. \& Lampel, J. (2005). Safari en pays stratégie. L'exploration des grands courants de la pensée stratégique. Pearson - Village Mondial : Eyrolles.

Paquette, G. (2002). L'ingénierie pédagogique : pour construire l'apprentissage en réseau. DesLibris : Books collection. Presses de l'Université du Québec.

Paquette, G. \& Léonard, M. (2013. Mars). Modèles et métadonnées pour les scénarios pédagogiques. Rapport Technique. Groupe de travail Québécois sur les normes et standards en TI pour l'apprentissage, l'éducation et la formation.

Pernin, J.-P. \& Godinet, H. (2006). Actes du colloque « Scénariser l'enseignement et l'apprentissage : une nouvelle compétence pour le praticien? », Lyon : INRP.

Pernin, J.P. \& Lejeune A. (2004). Dispositifs d'apprentissage instrumentes par les technologies : vers une ingénierie centrée sur les scénarios. Dans Actes du colloque TICE 2004, Compiègne, 407-414.

Schneider, D., Class, B., Frete, C., Girardin, F., Lombard, F., Morand. S. \& Synteta P. (2003). Conception et implémentation de scénarios pédagogiques riches avec des portails communautaires. Colloque de Guéret 4-6 Juin 2003.

Villiot-Leclercq, E. (2007). Modèle de soutien à l'élaboration et à la réutilisation de scénarios pédagogiques (Université Joseph Fourier, Grenoble; Université de Montréal). Repéré à https://tel.archivesouvertes.fr/tel-00156604 